ESAIM: PROCEEDINGS, November 2002, Vol.12, 108-114

M.Thiriet, Editor

\title{
MODELING AND SIMULATION OF THE FORMATION OF VASCULAR NETWORKS
}

\author{
DAPHne MANoussaki ${ }^{1}$
}

\begin{abstract}
The formation of blood vessels is driven by chemical and mechanical interactions between the cells and their extracellular matrix. New vasculature can emerge in the presence of angiogenic factors or as a result of cell traction forces onto the extracellular matrix. The respective contribution of chemical or mechanical events during angiogenesis is still unclear.

We present a mathematical model that incorporates such interactions and examine the conditions necessary for the formation of pattern. The model treats the extracellular matrix as a linear viscoelastic material and cellular movement either as a process influenced by the local strain field or as a chemotactic movement, in response to chemical gradients.
\end{abstract}

\section{INTRODUCTION}

New blood vessels can form during development by the reorganization of precursors to endothelial cells into a vascular network, a process termed vasculogenesis, or by sprouting of endothelial cells from existing vasculature (angiogenesis) [13,14]. Physiologic growth and remodelling of blood vessels stops in adults except for limited cases, such as wound healing and during the female reproduction cycle. Vessel growth can occur also in pathological cases, for example in tumor vascularization, which is required for tumor growth and metastasis [6]. Recent research efforts to control tumor growth via anti-angiogenesis drugs, an idea first proposed by J. Folkman in the early 70's [5], has brought a lot of attention into understanding angiogenesis.

Angiogenesis starts when endothelial cells in the lining of a blood vessel, in response to an angiogenic stimulus, degrade the adjacent collagen matrix and gradually sprout in the direction of the angiogenic stimulus. Known angiogenic factors include VEGF (vascular endothelial growth factor), FGFb (fibroblast growth factor b), or TAF (tumour angiogenesis factor, in the case of an angiogenic response elicited by a tumor).

The ECM plays an important role in the movement of cells towards the angiogenic stimulus: it provides molecules for cellular adhesion onto the matrix as well a scaffold necessary for cell spreading, cell migration and morphogenesis $[9,15]$. The cells can also alter the ECM by producing or degrading it, and deform it by applying mechanical forces to it and therefore cells can change the environment within which they migrate. This results to an active interaction between cells and their ECM, which is thought to be a key interaction during vasculogenesis $[15,16]$. In vitro models of vasculogenesis indicate that when endothelial cells are seeded onto a plane of Matrigel (gel-like collagen matrix), they attach onto it, pull it and reorganize it into a network of fibrous lines that tesselate the plane. These matrical lines are used by the cells as a scaffold for their migration. The cells fill the lines eventually forming a network of cellular cords [15]. During development, subsequent remodelling of the vascular plexus depends on cellular proliferation and further response of the cells to chemical and mechanical stimuli.

1 Department of Applied Mathematics, University of Crete, 71409 Heraklion, Greece, daphne@tem.uoc.gr

(C) EDP Sciences, SMAI 2003 
The relative importance of each of the type of interactions is still unclear. Mathematical modeling can describe different possible interactions in a cell - ECM environment and can help in testing the pattern forming capabilities under different hypotheses. Here, we present a mathematical model for the formation of blood vessels that is based on the Murray-Oster mechanochemical theory and takes into consideration the mechanical interaction between the cells and the ECM and can address cellular events such as cell proliferation, cell death, cell migration, cellular response to different chemical and mechanical events $[1,8,12]$.

\section{Mathematical Model}

The formation of pattern will be quantified in terms of changes in the local cell density, amount of ECM and ECM deformation. We use $n(\mathbf{x}, t)$ to denote the density of cells at time $t$ and on a point $\mathbf{x}$ in space. Cells apply forces to the ECM, so that each point of the ECM gets displaced by $\mathbf{u}=\left(u_{1}, u_{2}\right)$ from its original location $\mathbf{x}_{0}$, where $\mathbf{u}=\mathbf{x}(t)-\mathbf{x}_{0}$. These displacements create a strain field $\epsilon$ (approximated for small strains by $\left.\epsilon=\frac{1}{2}\left(\nabla \mathbf{u}+\nabla \mathbf{u}^{T}\right)\right)$ which can affect cellular movement. Cells, moreover, will respond to gradients of angiogenic factors and will move up such gradients. The chemical concentration we will denote by $c(\mathbf{x}, t)$, while the amount of ECM by $\rho(\mathbf{u}, t)$.

\subsection{Endothelial Cell Conservation Equation}

Local changes in cell density will take place as cells proliferate, die or move to neighbouring locations:

$$
\frac{\partial n}{\partial t}+\nabla \cdot J=r n\left(1-\frac{n}{N}\right)
$$

where the right hand term assumes a logistic model for the proliferation of cells, and the intrinsic growth rate of the cells $r$ can depend on different factors, such as growth factors and the local strain of the matrix $r=r(c, \epsilon)$.

$J$ denotes the cellular flux. Cells will migrate if they find themselves in areas of aligned ECM fibers [15], they will move up gradients of angiogenic factors, or will move passively, with velocity $\mathbf{v}=\frac{d}{d t} \mathbf{u}$, if they adhere onto a deforming matrix.

$$
\begin{aligned}
J & =-\nabla \cdot(D(\epsilon) n)+n \chi(c) \nabla c+n \frac{d u}{d t} \\
\text { Flux } & =\text { strain-dependent movement }
\end{aligned}
$$

The collagen fibre orientation, which affects cellular movement, is assumed to be a random movement, biased towards areas of principal strain and is modeled by the diffusion tensor

$$
D_{1}\left(\begin{array}{cc}
2+\epsilon_{11}-\epsilon_{22} & \epsilon_{12}+\epsilon_{21} \\
\epsilon_{12}+\epsilon_{21} & 2+\epsilon_{22}-\epsilon_{11}
\end{array}\right)
$$

(see [11]). The term $\chi(c)=\frac{\chi_{0}}{1+\beta c}$ is a form that is presented in [4] and it assumes that chemical gradients take effect when the chemical concentration is low; for high concentrations the corresponding receptors saturate and cannot feel the gradient.

\subsection{Matrix Conservation Equation}

In development the collagen matrix is produced by cells, and it is subsequently reorganized by them, as cells pull on it or migrate on it. In vitro there is often no significant production or degradation of matrix, and so we assume that local changes in matrix thickness occur just because the cells rearrange it on the culture dish:

$$
\frac{\partial \rho}{\partial t}+\nabla \cdot\left(\frac{d u}{d t} n\right)=0
$$


In considering the above form we shall assume that in general matrix production or degradation plays a less important role in the patterning process.

\subsection{Force Balance Equation}

The matrix reorganizes as a result of the traction forces that the cells exert onto it. We assume that total traction exerted by the cells is proportional to the cell density

$$
\sigma_{\text {cell }}=\tau n
$$

where $\tau$ is the intrinsic traction per cell, and it can depend on factors such as the local cell density, chemical stimuli, chemical gradients, local strain. Here, we assume that $\tau$ is isotropic and that it saturates at high cell densities, so that $\tau=\tau(n)=\tau_{0} \frac{1}{1+\alpha n^{2}} I$. We assume that the matrix responds to the traction forces as a linear viscoelastic material (Voigt body), so that the stress developed in the matrix when a strain $\epsilon$ is applied, is

$$
\sigma_{\text {matrix }}=\mu_{1} \epsilon_{t}+\mu_{2} \theta_{t} I+\frac{E}{\nu+1}\left(\epsilon+\frac{\nu}{1-2 \nu} \theta I\right)
$$

Additionally, the resistance to the movement of the matrix across the domain is modeled as a viscous drag

$$
F_{d r a g}=-s \frac{1}{\rho} \frac{d u}{d t}
$$

Since cells move distances of the order of a few microns to 100-200 microns at best over many hours, inertial effects are neglected and the forces between cells and the ECM are assumed to be approximately in balance:

$$
\begin{array}{r}
\nabla \cdot\left(\sigma_{\text {cell }}+\sigma_{\text {matrix }}\right)+F_{\text {drag }}=0 \\
\nabla \cdot\left(\tau_{0} \frac{1}{1+\alpha n^{2}} I+\mu_{1} \epsilon_{t}+\mu_{2} \theta_{t} I+\frac{E}{\nu+1}\left(\epsilon+\frac{\nu}{1-2 \nu} \theta I\right)\right)-s \frac{1}{\rho} \frac{d u}{d t}=0 .
\end{array}
$$

\subsection{Endothelial Activators}

Presence of endothelial cell activators are an integral part of cell activity and in many cases, for example in tumor angiogenesis, formation of capillary sprouts are considered to be up gradients of tumor angiogenesis factor (TAF), which is also uptaken by the cells $[3,7]$, or vascular endothelial growth factor (VEGF).

Here, we assume that such an angiogenic factor $c(\mathbf{x}, t)$ is uptaken by the endothelial cells and diffuses. In the model allow also for its potential secretion by the endothelial cells:

$$
\begin{aligned}
& \frac{\partial c}{\partial t}=D_{2} \nabla^{2} c \quad+\gamma n \quad-\delta \frac{n c}{K_{m}+c} \\
& \text { diffusion }+ \text { production by cells - uptake by cells }
\end{aligned}
$$

where $\gamma$ is the rate of production of $c$ by the cells, while the uptake is governed by Michaelis - Menten kinetics [4].

\section{AnAlysis}

During vasculogenesis, cells that are initially distributed throughout the domain of concern, reorganize themselves into networks. We model the initial condition as an almost uniform distribution of cells, matrix, chemical density, throughout the domain.

Through linear stability analysis we can derive the conditions under which a small perturbation (from the uniform state) in either the chemical concentration, or cell / matrix density or strain will give rise to pattern. We therefore assume that the problem variables are of the form 


$$
(n, \rho, \mathbf{u}, c)=\left(n_{0}, \rho_{0}, \mathbf{u}_{0}, c_{0}\right)+\left(n^{*}, \rho^{*}, \mathbf{u}^{*}, c^{*}\right) e^{\sigma t+i \mathbf{k} \mathbf{x}}
$$

where the asteriscs denote small quantities, i.e. we assume the variables near the uniform steady state $\left(n_{0}, \rho_{0}, \mathbf{u}_{0}, c_{0}\right)$ will grow or decay exponentially.

Substituting the above into the equations we can derive the dispersion relation, which associates different wavenumbers $\mathbf{k}$ with a growth rate $\sigma(\mathbf{k})$. Positive growth rates indicate that the initial small perturbation has the potential to grow and give rise to pattern. We get the following characteristic equation:

$$
\sigma\left(\frac{\delta}{K_{m}}\left(1-\frac{\gamma}{\delta}\right)^{2}+D_{2} k^{2}+\sigma\right)\left(a\left(k^{2}\right) \sigma^{2}+b\left(k^{2}\right) \sigma+c\left(k^{2}\right)\right)=0
$$

where

$$
\begin{aligned}
a\left(k^{2}\right) & =\mu k^{2}+s \\
b\left(k^{2}\right) & =\mu D_{1} k^{4}+k^{2}\left(s D_{1}+r \mu+(1+\nu)-\tau_{1}\right)+s r \\
c\left(k^{2}\right) & =k^{4}\left(D_{1}(1+\nu)-\frac{1}{4} \tau_{1} D_{1}\right)+k^{2} r(1+\nu)
\end{aligned}
$$

from the roots of which we get an expression for the growth rate $\sigma(\mathbf{k})$ as a function of the model parameters. From the above characteristic equation we observe that no values for the chemical parameters can lead to positive growth rates and that emergence of pattern (under the current model assumptions) is regulated by the mechanical behavior of the model: if $\tau_{1}=0$ (no cell traction), the equation has no roots of positive real part. If we assume no cell proliferation $(r=0)$, as may be reasonable for processes that happen within less than 24 hours - a period comparable to the doubling rate of cells, we get, in dimensional terms, the following condition that leads to positive growth rates and potential for formation of pattern:

$$
\frac{\tau}{E}>\frac{1}{1-\nu^{2}} \frac{1}{n_{0}} \frac{\left(1+a n_{0}^{2}\right)^{2}}{1-\alpha n_{0}^{2}}
$$

where $E$ is the matrix stiffness, $\tau$ is the traction per cell, $\nu$ is the Poisson ratio, $n_{0}$ is the initial uniform cell density, and $\alpha$ determines the decrease in total cell traction upon confluence. The result suggests that pattern can form if the ratio of cell traction to matrix stiffness or initial cell density $n_{0}$ are sufficiently large.

\section{RESUlts}

Starting from a small perturbation about the uniform steady state, linear stability analysis predicts formation of pattern if cellular traction $\tau$ is sufficiently high or matrix stiffness $E$ sufficiently low. The kinetic parameters of chemical production or uptake do not contribute to a positive growth rate of an initial perturbation. We do not mean that chemotaxis is not important. It plays a key role in the sprouting of endothelial cells from extant vasculature and its role has been discussed in mathematical models of tumor angiogenesis where capillary sprouts were shown to grow towards a source of chemoattractant $[2,4]$. What our results suggest is that chemotaxis alone is not a sufficient force to stimulate formation of pattern if we start with a perturbation to a uniform initial steady state, a situation that is a closer model for vasculogenesis, rather than angiogenesis (sprouting).

Other models of angiogenesis also suggest that sprout development towards a source of chemoattractant cannot be resolved by assuming only chemotaxis of cells and that other mechanisms also come into effect (e.g. haptotaxis, [4], cellular inhibition [10], etc.). 


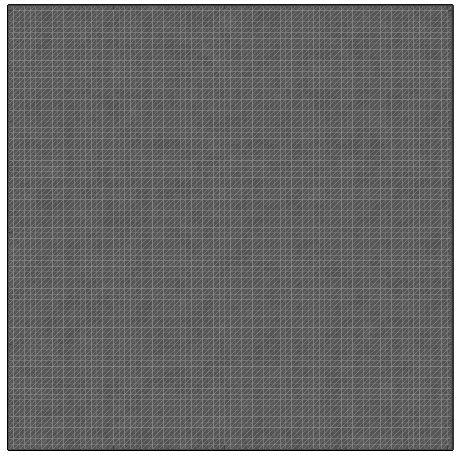

$\mathrm{t}=4$

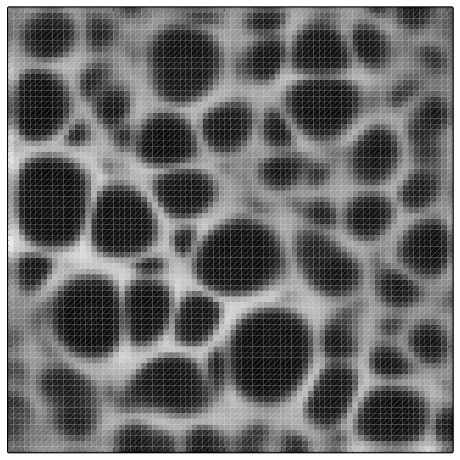

$\mathrm{t}=16$

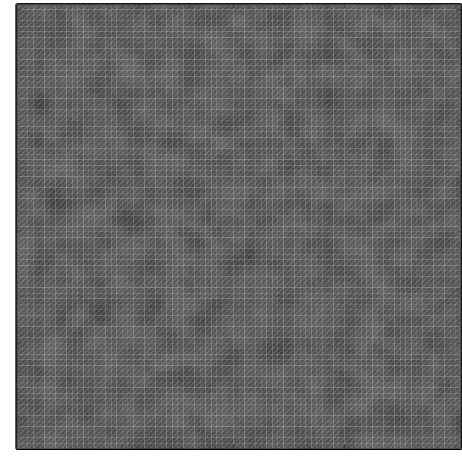

$\mathrm{t}=8$

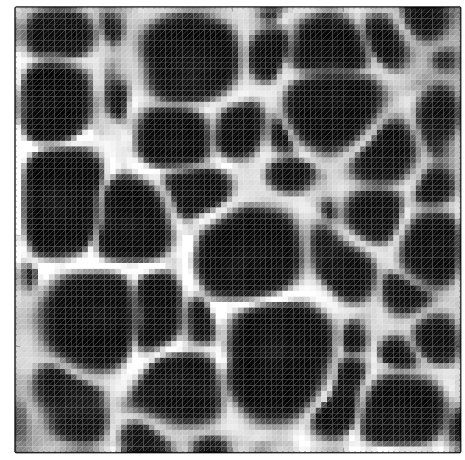

$\mathrm{t}=20$

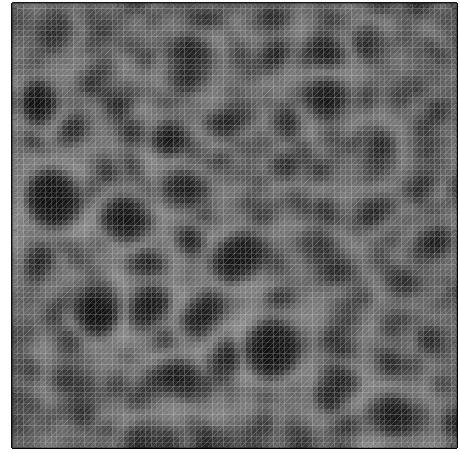

$\mathrm{t}=12$

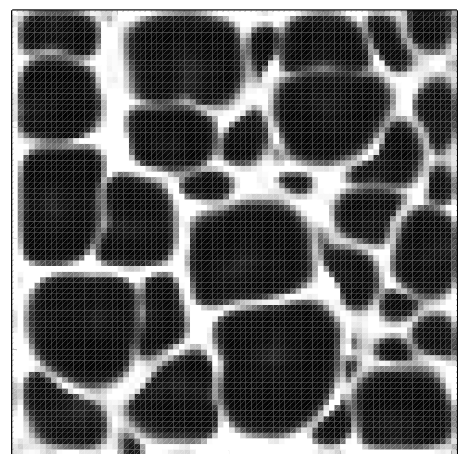

$\mathrm{t}=24$

FigURE 1. Numerical simulation of the rearrangement of cells into networks.

Initially cells are approximately uniform, but slowly areas of higher cell densities form (white) which exert traction onto the matrix and generate areas devoid of cells (black). Parameter values: $\tau=0.9$ dyne/cell, $D_{1}=10^{-11} \mathrm{~cm}^{2} / \mathrm{sec}, \nu=0.2, \mu_{1}=3 \times 10^{7}$ Poise, $E=20$ dyne $/ \mathrm{cm}^{2}, s=10^{10} \mathrm{dynes} \mathrm{sec} / \mathrm{cm}^{3}$. Simulations were performed on an $80 \times 80$ uniform grid, equations were discretized using finite differences. Zero cell-, ECM- and chemical flux is assumed through the boundary. Diffusion and stiff equations were treated using Crank-Nicholson discretization, advection terms using first order upwinding.

If we numerically simulate cellular movement in the absense of potential cell interactions with chemical gradients and no cell proliferation, we predict formation of network structures (fig. 1) that resemble cellular networks observed in in vitro experiments as well as examples of early planar vasculature. In the presence of a chemoattractant source, cells move up the gradient of a chemoattractant (fig. 2), and cell traction accelerates the movement of the cell clusters towards each other.

The agreement of the networks predicted by our model with those of in vitro models of vasculogenesis suggest further research into the role of cell-ECM mechanical interactions together with chemotaxis during angiogenic sprout formation. 


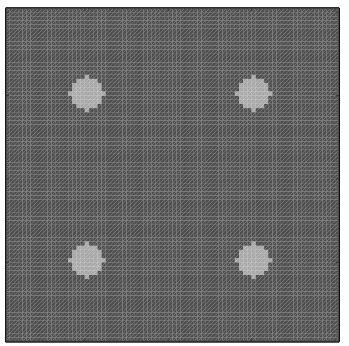

$t=1$

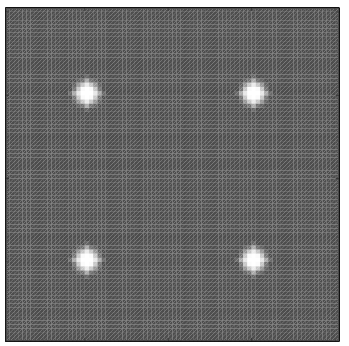

$t=1$

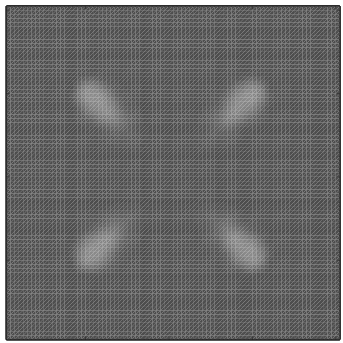

$t=15$

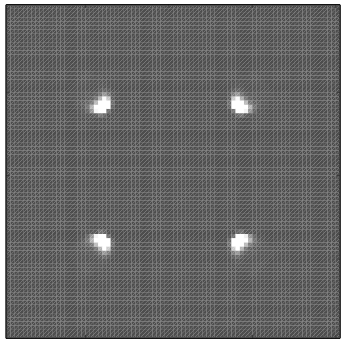

$\mathrm{t}=15$

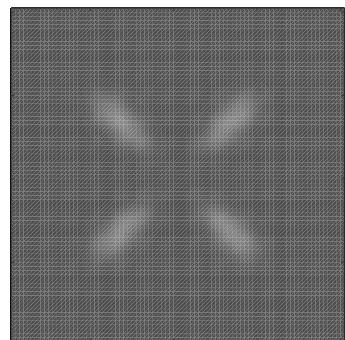

$t=30$

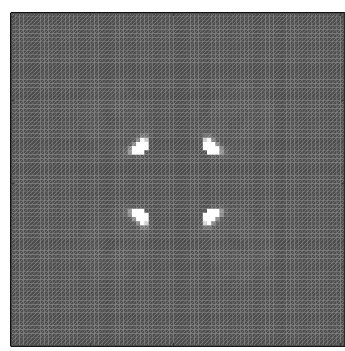

$\mathrm{t}=30$

FigURE 2.

If cells are made unable to pull (top figure), four cell clusters placed symmetrically around a central source of chemoattractant (positioned at the center of the domain), will move up gradients of increasing chemoattractant density. If cells can also exert traction (bottom figure), the cells will form more concentrated clusters and will move faster towards the chemoattractant source. In the absence of chemoattractant, cell clusters will move very little or not at all, depending on the distance between neighbouring clusters (not shown).

\section{REFERENCES}

[1] J.D. Murray ., G.F. Oster, and A.K. Harris. A mechanical model for mesenchymal morphogenesis. Journal of Mathematical Biology, 17:125-129, 1983.

[2] A.R. Anderson and M.A. Chaplain. Continuous and discrete mathematical models of tumor-induced angiogenesis. Bulletin of Mathematical Biology, 60:857-900, 1998.

[3] D.H. Ausprunk and J. Folkman. Migration and proliferation of endothelial cells in preformed and newly formed blood vessels during tumour angiogenesis. Microvascular Research, 14:53-65, 1977.

[4] M.A. Chaplain and A.R. Anderson. Mathematical modelling, simulation and prediction of tumour-induced angiogenesis. Invasion Metastasis, 16(4-5):222-234, 1996.

[5] J. Folkman. Tumor angiogenesis: therapeutic implications. New England Journal of Medicine, 285:1182-1186, 1971.

[6] M.A. Gimborne, R. S. Cotran, S.B. Leapman, and J. Folkman. Tumor growth and neovascularization: An experimental model using the rabbit cornea. J. Natl. Cancer Inst., 52:413-427, 1974.

[7] D. Hanahan. Signaling vascular morphogenesis and maintenance. Science, 227:48-50, 1997.

[8] D. Manoussaki, S.R. Lubkin, R.B. Vernon, and J.D. Murray. A mechanical model for the formation of vascular networks in vitro. Acta Biotheoretica, 44(3-4):271-282, 1996.

[9] R.R. Markwald, T.P. Fitzharris, D.L. Bolender, and D.H. Bernanke. Sturctural analysis of cell:matrix association during the morphogenesis of atrioventricular cushion tissue. Developmental Biology, 69(2):634-54, 1979.

[10] H. Meinhardt. Models for the formation of netline structures. In Charles D. Little, Vladimir Mironov, and E. Helene Sage, editors, Vascular Morphogenesis: In Vivo, In Vitro, In Mente, chapter 3.1, pages 147-172. Birkauser, Boston, MA, 1998. 
[11] J.D. Murray, D. Manoussaki, S.R. Lubkin, and R.B. Vernon. A mechanical theory of in vitro vascular network formation. In Charles D. Little, Vladimir Mironov, and E. Helene Sage, editors, Vascular Morphogenesis: In Vivo, In Vitro, In Mente, chapter 3.2, pages 173-188. Birkauser, Boston, MA, 1998.

[12] G.F. Oster, J.D. Murray, and A.K. Harris. Mechanical aspects of mesenchymal morphogenesis. Journal of embryology and experimental morphology, 78:83-125, 1983.

[13] L. Pardanaud, F. Yassine, and F. Dieterlen-Lievre. Relationship between vasculogenesis, angiogenesis and haemopoiesis during avian ontogeny. Development, 105:473-485, 1989.

[14] W. Risau, H. Sariola, H.G. Zerwes, J. Sasse, P. Ekblom, R. Kemler, and T. Doetschmann. Vasculogenesis and angiogenesis in embryonic-system-cell-derived embryoid bodies. Development, 102:471-478, 1988.

[15] R.B. Vernon, J.C. Angello, M.L. Iruela-Arispe, T.F. Lane, and E.H. Sage. Reorganization of basement membrane matrices by cellular traction promotes the formation of cellular networks in vitro. Laboratory Investigation, 66(5):536-547, 1992.

[16] R.B. Vernon and E.H. Sage. Between molecules and morphology: Extracellular matrix and the creation of vascular form. American Journal of Pathology, 147:873-883, 1995. 